\title{
VALUACIÓN DE LAS EXPECTATIVAS DEL SECTOR BANCARIO EN MÉXICO
}

\section{Expectations of the Mexican Banking Sector Valuation}

\author{
| Ricardo Cristhian Morales Pelagio ${ }^{1}$
}

\section{RESUMEN}

El presente trabajo tiene por objeto analizar qué genera o maximiza el valor de mercado de los grupos financieros que cotizan en la Bolsa Mexicana de Valores (BMV), si un incremento en el crecimiento o un aumento en su rentabilidad. Para ello, se considera el valor terminal o perpetuidad desarrollado por Gordon y Shapiro, utilizado en la metodología de valuación por flujos de dividendos descontados, así como información financiera del periodo 2014 al 2018. Además, se aplica el análisis propuesto por Mass para evaluar el potencial del crecimiento y el rendimiento. Se determinó que el valor generado por el crecimiento es mayor que el obtenido por el incremento en la rentabilidad en las cinco instituciones financieras analizadas. Los resultados implicarían que todavía existe un crecimiento potencial y que las instituciones financieras aún no se encuentran totalmente maduras o desarrolladas y/o en un mercado maduro o desarrollado, por lo que las estrategias vía Fintech para crecer serían más valoradas por el mercado.

Palabras clave: crecimiento, rentabilidad, valor del capital de accionistas, valor relativo del crecimiento.

Clasificación ]EL: C65, G11, N26.

\footnotetext{
${ }^{1}$ Doctor en Ciencias de la Administración por la FCA-UNAM, y Profesor de la División de Investigación de la misma Facultad. Correo electrónico: pelagioricardo@hotmail.com

Fecha de recepción: 10/02/2020 • Fecha de aceptación: 25/06/2020
} 


\section{ABSTRACT}

The present work has the objective of analyzing what generates or maximizes the market value of the financial groups listed on the Mexican Stock Exchange (BMV), given an increase in either growth or profitability. For this, the terminal value or perpetuity developed by Gordon and Shapiro is considered and used in the valuation methodology for discounted dividend flows, as well as financial information for the period 2014 to 2018. In addition, the analysis proposed by Mass is applied to assess the potential for growth and yield. It is determined that the value generated by the growth is greater than that obtained by the increase in profitability in the five financial institutions analyzed. The results would still imply that potential growth exists, that the financial institutions are not yet fully mature or developed and/or not operate in a mature or developed market, so that Fintech strategies to promote growth would be better valued by the market.

Keywords: growth, profitability, Equity value, relative value of growth JEL Classification: C65, G11, N26.

\section{Introducción}

$\mathrm{E}$ n la actualidad existen organizaciones que tienen el problema de identificar cómo generar o crear valor, lo que propicia un desempeño deficiente que impacta en el desarrollo financiero de la empresa y en la generación de valor para el accionista. Existen casos en que los directivos no tienen un entendimiento claro de lo que es generar valor y tienen la creencia que se genera valor siempre que una organización crece, ya sea en ingresos, utilidades o activos. Lo anterior, trae como consecuencia que busquen crecer a las mayores tasas posibles descuidando la rentabilidad o deteriorando los márgenes de ganancia (Entzana, 2016).

Los directivos de empresas se enfrentan al reto de lograr crecimiento y mantener rentabilidad a la vez; inclusive hay posturas que contraponen entre qué es más importante, si generar crecimiento de utilidades o priorizar el margen de rentabilidad. Bajo un contexto idóneo deberían darse las dos cosas, crecimiento de ingresos y utilidades con mantenimiento o crecimiento de la rentabilidad; pero la realidad es otra: la premisa de crecer y tener un margen de rentabilidad óptimo a la vez es difícil. Cabe destacar que a largo plazo los dos son importantes y deben de considerarse para establecer la base para generar valor, ya que si el re- 
sultado de la empresa es crecer y sistemáticamente se va disminuyendo el margen de rentabilidad se destruye valor a largo plazo e igualmente se incrementa la rentabilidad, pero no se tiene crecimiento.

El problema es que, con el objetivo de crear y maximizar el valor a largo plazo de forma sostenible, por un lado no saben o no identifican qué es más importante o fundamental en la toma de decisiones en el corto plazo, si el crecimiento o la rentabilidad. y por otro lado qué tanto del margen de rentabilidad es aceptable sacrificar o llegar a disminuir por crecer. Esta situación la sufren todas las empresas, independientemente del sector o tamaño. Sin embargo, la respuesta no es la misma para cada empresa, sino que está determinada en función de las circunstancias o características bajo las cuales se encuentra cada empresa o sector.

Es decir, la problemática radica en que muchas veces las empresas desconocen o se equivocan en cuál es la acción inmediata o urgente a atender en el presente, si crecimiento o rentabilidad y por ende toman las decisiones incorrectas para orientar a la empresa hacia la generación o creación de valor para el accionista. También, en ocasiones siguen estrategias de crecimiento, pero sin conocer y estimar hasta qué grado o nivel es aceptable mantener el margen de rentabilidad. Es decir, hasta qué punto, se genera más valor manteniendo o incrementando la rentabilidad en lugar del crecimiento (Entzana, 2016).

Se debe de identificar qué decisiones son las correctas acerca de cómo generar valor, qué estrategia inmediata es la más importante para maximizar el valor del capital de los accionistas. Por un lado, a veces se continúa con planes de expansión y crecimiento exponiéndose al riesgo de destruir valor o incluso de llevar a la quiebra a la empresa. En contraposición, muchas veces por ahorrar costos y mejorar el margen de rentabilidad o ganancia frenan el crecimiento de la empresa en detrimento de la generación de valor a futuro.

Específicamente en el sector bancario en México, a la luz del desarrollo de la digitalización no sólo de sus procesos, sino de la expansión de oferta y venta de sus productos y servicios financieros a través de las Fintech (tecnologías de operación y servicios financieros por internet), se plantea la interrogante de qué expectativa más evidente presenta el mercado, si un mayor valor por el crecimiento o por la rentabilidad que le puede dar esta estrategia basada en las tecnologías financieras y digitalización de sus productos y servicios financieros. La digitalización en la banca a través de las Fintech en el largo plazo, cumple con la estrategia idónea ya que permitiría maximizar el valor a través tanto del crecimiento como de la rentabilidad (al igual que en algunas estrategias de adquisición y fusión empresarial). 
El objetivo de este trabajo es: analizar qué estrategia, si de crecimiento o de rentabilidad, maximiza el valor de mercado de los grupos financieros que cotizan en la Bolsa Mexicana de Valores (BMV). Es decir, surge la pregunta ¿qué estrategia inmediata se debe implementar a través de este tipo de tecnología: una que haga más énfasis en sus procesos para incrementar rentabilidad de operación, o una que se enfoque más en buscar un mayor crecimiento a través de una mayor oferta y distribución de sus productos y servicios financieros con las Fintech? En otras palabras, descubrir qué maximizará el valor de los bancos en México de forma presente o a corto plazo, crecimiento o rentabilidad, de acuerdo con las expectativas del mercado.

Se tomó una muestra de 5 empresas financieras que cotizan actualmente en la Bolsa Mexicana de Valores y realizan actividades de banca y concernientes a un grupo financiero. A través del valor terminal de acuerdo con la metodología de flujos de efectivo descontados, considerando los dividendos como flujo de efectivo, se utilizó el modelo de Mass (2005) cuya métrica estimaba el valor relativo del crecimiento, es decir, si generaba más valor el crecimiento o la rentabilidad.

La hipótesis que se plantea es que el valor será maximizado con una estrategia de crecimiento más que de rentabilidad, dada la oportunidad de una significativa bancarización a través de una mayor oferta de productos y servicios a través de la digitalización; por lo que es posible llegar a más mercados y consumidores de una forma más rápida y masiva.

Además de esta introducción, el trabajo se encuentra integrado por tres secciones más. En la sección siguiente se desarrolla una breve revisión de la literatura acerca del valor y la valuación de las empresas; posteriormente se aborda en la metodología el modelo de valuación de empresas por flujos de efectivo descontados; por último, se presentan los resultados y las conclusiones con base a la evidencia encontrada en las empresas de la muestra.

\section{Revisión de la literatura}

Una de las metodologías ampliamente aceptadas para estimar el valor de la empresa y capital de accionistas es la de los flujos de efectivos descontados. Fernández (2013) afirma que el método más apropiado para valuar una empresa es mediante el descuento de sus flujos de efectivo esperados a futuro, ya que el valor de las acciones de una empresa proviene de la capacidad de la misma de generar 
flujos de efectivo libres para los accionistas. Al respecto, Copeland, Kollery Murrin (2000) aplicaron la metodología de valuación por flujos de efectivo descontados a 31 empresas en los Estados Unidos y encontraron una fuerte correlación entre los flujos de efectivo y los valores de mercado de las empresas.

En el medio financiero profesional, la mayoría de analistas de grupos financieros tanto nacionales como internacionales ocupan principalmente esta metodología de valuación junto con la de múltiplos, siendo un referente también para otras empresas consultoras, de asesoría financiera y valuación de empresas en México. Cain y Dennis (2008), investigaron cuales son los métodos para valuar empresas que utilizan los bancos de inversión y encontraron que el 75\% de los analistas de bancos ocupan el método de flujos descontados, seguido del de múltiplos de empresas públicas y de transacción de empresas similares. En México, Saavedra (2002) aplicó diferentes metodologías de valuación a 71 empresas y encontró que el valor estimado por la metodología de flujos es el que más se acercaba al precio de mercado de las empresas. Por su parte, Adam (2005) demuestra la relación entre el análisis fundamental con el rendimiento bursátil.

Al respecto de dicha concepción de valor, Mill (1848) y Marshall (1890) fueron de los primeros autores que se refirieron al valor considerando un enfoque financiero. Mill (1848) argumentaba que las ganancias brutas del capital, son las ganancias devueltas a los que suministran fondos para la producción y tienen que ofrecer un equivalente suficiente para compensar la abstinencia, y el riesgo al que se somete el empresario o inversionista. Por su parte Marshall (1890) establecía que el empresario debía obtener los rendimientos del capital invertido, incluyendo el fondo de comercio y de su propia capacidad, debiéndose considerar esto como un costo de oportunidad; acuñó el término de beneficio económico el cual representa el valor generado en función de la rentabilidad lograda por encima del costo de oportunidad o la tasa mínima requerida por el accionista. Derivado del concepto de beneficio económico, surge el EVA ${ }^{\circledast}$ (Economic Value Added) el cual fue creado por Stern Steward \& Co., el cual busca estimar el valor generado en un periodo por una empresa.

Sobre la generación de valor de la empresa, Drucker (1995) afirma que mientras un negocio tenga un rendimiento inferior a su costo de capital, operara con pérdidas. No importa que pague impuestos como si tuviera una ganancia real. La empresa aún deja un beneficio económico menor a los recursos que devora, mientras esto suceda no crea riqueza, la destruye. Friedman (1970) expresa que 
la dirección o gerencia de una empresa tiene como principal objetivo maximizar el valor de la misma para sus accionistas o propietarios.

Rappaport afirma que en una economía de mercado, la única responsabilidad de un negocio es crear valor para el accionista y hacerlo de un modo legal y con integridad. Por otro lado, Copeland, Koller y Murrin (2000) hacen referencia a la importancia de que los directivos se centren en la generación de valor para los accionistas, pues si bien es cierto, de esta manera se crearán empresas más sanas que generaran a su vez economías solidas lo que se traduce en mejores niveles de vida y oportunidades en general.

Mass(2005) realizó un análisis de las principales empresas estadounidenses para determinar qué estrategia genera mayor valor para el accionista, crecimiento en ventas o rentabilidad, encontrando resultados mixtos, y llegando a la conclusión de que muchas de ellas no van por la senda correcta.

Con respecto a la estimación del valor de la empresa por flujos de efectivo, WiIliams (1938), desarrolló la fórmula que hasta hoy día se conoce como el valor presente de una perpetuidad creciente, la cual estima el valor presente de una serie de pagos periódicos a una tasa de crecimiento constante por un tiempo indefinido. Posteriormente, Gordon y Shapiro (1956), aplicaron la fórmula desarrollada por Williams para el caso de la determinación del precio de una acción en función a los dividendos estimados que pagaría la acción. Con la información de los dividendos pagados a futuro y considerando un crecimiento perpetuo de ellos después de un periodo de tiempo Gordon y Shapiro (1956) estimaron el valor presente de una acción siendo la primera estimación del valor del capital accionario de una empresa por un método de flujos descontados.

Posteriormente, Myers (1974) presenta su modelo de valuación por flujos de efectivo Adjusted Present Value (APV), el cual establece que el valor de la empresa apalancada es igual al valor de la empresa sin deuda más el valor presente del ahorro de impuestos debido al pago de intereses. Arditti y Levy (1977) analizan la valuación de empresas por flujos considerando el flujo de efectivo disponible para el capital y el flujo de efectivo libre para la empresa, y consideran que el primero de estos flujos es mejor para estimar el valor de la empresa, mismo que debe descontarse al costo de capital antes de impuestos. Concluyen que el valor de la empresa es distinto de acuerdo con el flujo de efectivo que se utilice.

Fuller y Hsia (1984), desarrollaron el modelo $\mathrm{H}$ en el cual se determina el modelo de patrón de crecimiento de los dividendos, estableciendo un periodo de crecimiento cada vez menor hasta llegar un crecimiento constante y así deter- 
minar después de un periodo de tiempo el valor de los dividendos a crecimiento perpetuo.

El enfoque anterior se vio ampliado no sólo a los dividendos sino también a los demás flujos que generaba la empresa y que podían ser susceptibles de descontarse para obtener el valor de la misma, Pinto, Henry y Stowe (2007), establecieron tres modelos de patrón de crecimiento en la empresa, aplicado no sólo a los dividendos sino a las ventas, las utilidades y los flujos de efectivo. Un modelo de crecimiento de una etapa en el que la empresa ya estaba en un crecimiento constante, el modelo de tres etapas en el que la empresa experimentaba en la primera etapa un alto crecimiento; en la segunda etapa una tasa de crecimiento cada vez menor hasta llegar a la tercera etapa que es la de crecimiento constante a largo plazo. También se refirieron al modelo de dos etapas similar al de Fuller y Hsia.

Por su parte, Damodaran (2006) plantea las principales decisiones en finanzas corporativas y su interrelación, así como la política que estas deben seguir para la generación y maximización del valor de la empresa. Desarrolla las metodologías de valuación por el flujo de efectivo libre para la empresa y el flujo libre para el accionista y establece las ventajas y conveniencias de la utilización de este método. Fernández (2013) también argumenta la utilización del método de flujos de efectivos descontados como el mejor para estimar el valor de la empresa y sugiere cambios en el cálculo del valor terminal de la empresa.

\section{Metodología}

\subsection{Valuación por flujos de efectivos descontados.}

Según la metodología de flujos de efectivos descontados, el valor de un activo está en función de su capacidad de generar flujos libres de efectivo a futuro, es decir, el precio que se paga por un activo o empresa es el valor presente de los flujos de efectivos descontadas a una tasa de interés; en función del tipo de flujo de efectivo y la tasa de descuento, se considera si se está determinando el valor de la empresa o del capital accionario (ver cuadro 1). 
Cuadro 1. Flujos de EFeCtivo, tASAS DE DESCUENTO Y VALOR ESTIMAdo

\begin{tabular}{lll}
\hline Flujo & Tasa de descuento & Valor \\
\hline Dividendos & Ke & Capital de accionistas \\
\hline Flujo de Efectivo Libre para los Accionistas (FCFE) & Ke & Capital de accionistas \\
\hline Flujo de Efectivo Libre para la Empresa (FCFF) & WACC & Empresa \\
\hline
\end{tabular}

Fuente: Software Corporate Finance 101, de Zoologic Inc.

Dada la naturaleza del giro del negocio de las empresas o instituciones financieras, se deberá utilizar los dividendos como flujo de efectivo a descontar para determinar el valor del capital de accionistas. Dicho flujo se descuenta a la tasa del costo del capital accionario (Ke):

$$
\text { Precio }=\frac{D i v_{1}}{(1+K e)^{1}}+\frac{D i v_{2}}{(1+K e)^{2}}+\cdots \frac{D i v_{n}}{(1+K e)^{n}}+\frac{\frac{D i v_{n} *(1+g)}{K e-g}}{(1+K e)^{n}}
$$

El costo del capital accionario se refiere a la tasa de rendimiento mínima exigida por los accionistas o socios sobre los recursos que aportaron para financiar los proyectos y actividades de la empresa; dicha tasa debe de reflejar el riesgo de los proyectos de la empresa, así como el costo de oportunidad del capital en el periodo. Para su determinación se ocupa algún modelo de riesgo financiero, siendo en el presente caso el modelo de valuación de activos financieros CAPM, que se determina mediante la siguiente fórmula:

$$
K e=R f+B e t a *(R p)
$$

donde:

$K_{e}=$ rendimiento mínimo esperado por el accionista
$R_{f}=$ tasa de rendimiento para un activo libre de riesgo

$\beta_{R}=$ beta de la acción de la empresa

$R_{p}=$ premio de riesgo del mercado 
Nótese en la fórmula (1) que el último flujo a descontar para obtener el valor de la empresa incluye el valor terminal (VT), que significa el valor de los flujos de dividendos perpetuos a partir del último año estimado y representa el valor de la acción o capital accionario en el año n. La fórmula para su cálculo utilizada es la propuesta por Gordon y Shapiro (1956):

$$
V C A_{n}=V T=\frac{D i v_{n} *(1+g)}{K e-g}
$$

Donde g es la tasa de crecimiento a perpetuidad, $\operatorname{Div}_{n}$ es el último flujo de dividendos pronosticado, VCA es el valor del capital de accionistas y el Ke es el costo de capital accionario asumido en el periodo. Como se observa en la fórmula hay dos componentes fundamentales para la estimación del valor terminal y de la acción o capital accionario en el año n estimado. El primero es el último flujo de dividendos considerado puesto que en función de este se aplicara el crecimiento perpetuo de los dividendos. El segundo es la tasa de crecimiento a perpetuidad $g$ puesto que a mayor nivel de esta tasa se alcanzará un mayor valor de la empresa. A continuación, se utilizará esta fórmula para estimar el crecimiento implícito que considera el mercado para asignar el valor del capital accionario de cada institución financiera en el mercado.

\subsection{Estimación del crecimiento a perpetuidad implícito y el valor de la empresa}

Una vez vista la metodología de valuación por flujos y explicado el término de valor terminal, a partir de la fórmula (3.3) podemos estimar cuál es el crecimiento que el mercado accionario mexicano considera a largo plazo para los accionistas de las empresas. Como se definió, el valor terminal (VT) es el valor de los flujos de efectivo perpetuos a partir del último año considerado (n). Con base en ello y en la definición de que el valor del capital de accionistas es el valor de los flujos de dividendos futuros traído al día, podemos considerar el valor de dicho capital en el periodo $n$ mediante la fórmula (4):

$$
V T_{n}=\frac{D i v_{n} *(1+g)}{K e-g}=V C A
$$


Asumiendo que por valor del capital de accionistas (VCA) de la empresa se considera el que provee el mercado (capitalización bursátil) y que la información del Ke y Dividendos es la que tiene la empresa en el último año conocido, al despejar se puede calcular la variable g, expresada de la siguiente manera:

$$
g=\frac{(V C A * K e)-D i v_{n}}{D i v_{n}+V C A}
$$

Una vez conocido el valor del crecimiento a perpetuidad (g), se esw posible estimar el valor del capital de accionistas de la empresa producto de un incremento en la tasa de crecimiento considerada por el mercado; es decir el valor que hubiese tenido el capital de accionistas de la empresa por una unidad porcentual adicional de crecimiento:

$$
V C A_{n}^{\prime}=\frac{D i v_{n} *\left(1+g^{\prime}\right)}{K e-g^{\prime}}
$$

Donde g' es igual a la tasa g implícita considerada por el mercado más el incremento de un punto porcentual, y VCA' es el valor del capital de accionistas estimado en el periodo que hubiese tenido la empresa con ese mayor crecimiento a perpetuidad. Igualmente, si se considera un punto porcentual adicional en la tasa de rentabilidad, medida ésta por el margen neto, se tendría un mayor flujo de dividendos en el último periodo (Div' ${ }_{n}$ ) por lo que el valor del capital de accionistas de la empresa que hubiese sido se determinaría con:

$$
V C A_{n}^{\prime}=\frac{\operatorname{Div}_{n}^{\prime}(1+g)}{K e-g}
$$

Planteado lo anterior, se tomarán para la muestra de grupos financieros aqueIlas entidades que cotizan en la BMV, se estimará su tasa de crecimiento a perpetuidad implícita considerada por el mercado, tomando el valor de la empresa a finales del año 2018. Posteriormente se hará la prueba del cambio en el valor al modificar el crecimiento, ceteris paribu,s y luego cambiando el flujo de efectivo 
libre, ceteris paribus, al incrementarse un punto porcentual por arriba del margen neto.

\section{Resultados: estimación del crecimiento a perpetuidad y del valor que genera la rentabilidad y crecimiento en las empresas}

En el cuadro 2 se presentan la muestra de los grupos financieros que cotizan en la BMV considerados para el presente trabajo. De acuerdo a el Sistema de Clasificación de la Industria Norteamericana (NAICS), son empresas con actividades bursátiles, cambiarias y de inversión financiera. Al final se tuvo una muestra de cinco empresas y se excluyó a GFMultiva debido a la baja o nula bursatilidad que posee y la ausencia de pagos de dividendos en los últimos años.

CuAdRo 2. MUESTRA DE GRUPOS FINANCIEROS QUE COTIZAN EN LA BMV

\begin{tabular}{lll}
\hline No. & Nombre & Código \\
\hline 1 & Banco Del Bají & BBAJIOO \\
\hline 2 & GFBanorte & GFNORTEO \\
\hline 3 & GFInbursa & GFINBURO \\
\hline 4 & Regional & RA \\
\hline 5 & Santander Serfin GF & BSMXB \\
\hline
\end{tabular}

Fuente: elaboración propia con datos de Economática.

En el cuadro 3 se presenta el costo de capital de accionistas (Ke) a través del modelo de valuación de activos financieros (CAPM, por sus siglas en inglés). El coeficiente beta ajustado se calculó a partir de rendimientos semanales del periodo 2017-2018, a excepción de BBAJIOO que se calculó con rendimientos diarios de 2018 dada su baja bursatilidad; para la tasa libre de riesgo (Rf) se considera la tasa de 7.29 por ciento con base al promedio geométrico de las tasas del Bono $\mathrm{Ma}$ 10 años del periodo 2014-2018. Por su parte, para la prima de riesgo del mercado se consideró una tasa del 5.39 por ciento, determinada de acuerdo a las expecta- 
tivas del mercado accionario mexicano en función de su histórico de rendimientos y de los rendimientos de otros mercados.

CUADRO 3. BETA Y COSTO DE CAPITAL DE ACCIONISTAS DE LOS GRUPOS FINANCIEROS QUE COTIZAN EN LA BMV

\begin{tabular}{llll}
\hline No. & Nombre & Beta & Ke \\
\hline 1 & Banco Del Bajío & 1.033 & $12.86 \%$ \\
\hline 2 & GFBanorte & 1.27 & $14.12 \%$ \\
\hline 3 & GFInbursa & 0.75 & $11.31 \%$ \\
\hline 4 & Regional (Banregio) & 1.03 & $12.86 \%$ \\
\hline 5 & Santander Serfin GF & 1.09 & $13.15 \%$ \\
\hline
\end{tabular}

Fuente: elaborado con datos de Economática.

En el cuadro 4 se presentan los valores de mercado del capital de accionistas de las empresas abordadas en el trabajo; se tomaron los valores de capitalización bursátil del 30 de septiembre de 2018. La razón de tomar hasta el tercer trimestre y no el final del año fue para no sesgar o alterar el valor que les otorgaba el mercado antes de la incertidumbre generada por el cambio de gobierno federal en el 2018. Para el flujo de dividendos, se tomó el promedio de dividendos del periodo 2014 al 2018, con el objeto de no sobre o subvaluar el monto de dividendos por algún año anormal en algún lapso de los últimos años y estimar mejor el flujo de dividendos generado por la empresa.

CuAdro 4. VALOR DE MERCADO DEL CAPITAL DE LOS ACCIONISTAS Y DIVIDENDOS ESTIMADOS MILLONES DE PESOS

\begin{tabular}{llrc}
\hline No. & Nombre & \multicolumn{1}{c}{ Dividendos } & \multicolumn{1}{c}{ Valor Capital } \\
\hline 1 & Banco Del Bají & 607.5 & 54,951 \\
\hline 2 & GFBanorte & $7,088.6$ & 390,132 \\
\hline 3 & GFInbursa & $2,611.8$ & 195,009 \\
\hline 4 & Regional & 434.3 & 38,624 \\
\hline 5 & Santander Serfin GF & $9,532.2$ & 197,773 \\
\hline
\end{tabular}

Fuente: elaborado con datos de Economatica. 
Con la información anterior y utilizando la ecuación (5) planteada en la sección de metodología, se determina en términos nominales la tasa de crecimiento a perpetuidad (g) implícita de los dividendos que asume el mercado para justificar el valor del capital de accionistas, en función de la metodología de valuación de flujos descontados. En el cuadro 5 se observa cómo, salvo Santander y CFInbursa, los demás grupos financieros tienen altas tasas de crecimiento esperadas, de dos dígitos (o casi dos dígitos en el caso de Banco del Bajío), lo que evidencia altas expectativas del sector, comparado con el crecimiento de la economía en general, es decir del Producto Interno Bruto (PIB).

CUADRO 5. ESTIMACIÓN DE LA TASA DE CRECIMIENTO A PERPETUIDAD (C) IMPLÍCITA DE ACUERDO CON LOS DIVIDENDOS Y VALOR DE MERCADO DEL CAPITAL DE ACCIONISTAS

\begin{tabular}{llrrrl}
\hline No. & Nombre & Dividendos & Ke & Valor Capital & g implícita \\
\hline 1 & Banco Del Bajío & 607.5 & $12.86 \%$ & 54,951 & $11.63 \%$ \\
\hline 2 & GFBanorte & $7,088.6$ & $14.12 \%$ & 390,132 & $12.08 \%$ \\
\hline 3 & GFInbursa & $2,611.8$ & $11.31 \%$ & 195,009 & $9.84 \%$ \\
\hline 4 & Regional & 434.3 & $12.86 \%$ & 38,624 & $11.60 \%$ \\
\hline 5 & Santander Serfin GF & $9,532.2$ & $13.15 \%$ & 197,773 & $7.94 \%$ \\
\hline
\end{tabular}

Fuente: elaborado con datos de Economática.

En el cuadro 6 se observa el valor del capital de accionista estimado, considerando un incremento de uno por ciento en la tasa a perpetuidad de los dividendos, utilizando la ecuación (6) presentada en la metodología. A través de la metodología de flujos, es evidente cómo el mercado además de tener altas expectativas de crecimiento de los grupos financieros que cotizan en la BMV, también incrementa significativamente el valor del capital de accionistas ante un aumento de su tasa de crecimiento a perpetuidad. Destacan los casos de Banco del Bajío y Regional (antes Banregio) con incrementos del valor de alrededor de 400 por ciento, así como GFInbursa de más de 200 por ciento. 
CUADRO 6. ESTIMACIÓN DEL VALOR DEL CAPITAL DE ACCIONISTAS CONSIDERANDO UN INCREMENTO DEL 1 POR CIENTO EN LA TASA DE CRECIMIENTO

\begin{tabular}{llrrrr}
\hline No. & Nombre & Valor Capital & $\mathbf{g}+\mathbf{1 \%}$ & Valor Capital & \multicolumn{1}{c}{$\boldsymbol{\Delta \% \text { Valor Capital }}$} \\
\hline 1 & $\begin{array}{l}\text { Banco Del } \\
\text { Bají }\end{array}$ & 54,951 & $12.63 \%$ & $292,324.64$ & $431.97 \%$ \\
\hline 2 & GFBanorte & 390,132 & $13.08 \%$ & $773,371.71$ & $98.23 \%$ \\
\hline 3 & GFInbursa & 195,009 & $10.84 \%$ & $614,445.92$ & $215.09 \%$ \\
\hline 4 & Regional & 38,624 & $12.60 \%$ & $191,928.96$ & $396.92 \%$ \\
\hline 5 & $\begin{array}{l}\text { Santander } \\
\text { Serfin GF }\end{array}$ & 197,773 & $8.94 \%$ & $247,099.99$ & $24.94 \%$ \\
\hline
\end{tabular}

Fuente: elaborado con datos de Economática.

En el cuadro 7 se observa cómo el incremento del valor del capital de accionistas por un aumento del margen neto de los grupos financieros, implica menos del 10 por ciento de mayor valor, salvo el caso de Banco del Bajío. Para el caso de GFInbursa el incremento en la rentabilidad impacta poco más de un 2 por ciento en el valor del capital de accionistas. Es evidente que en este caso el crecimiento genera mayor impacto que la rentabilidad en el flujo de dividendos y el valor de los accionistas.

\section{CUADRO 7. ESTIMACIÓN DEL VALOR DEL CAPITAL DE ACCIONISTAS CONSIDERANDO UN INCREMENTO DEL 1 POR CIENTO EN SU MARGEN DE UTILIDAD NETA}

\begin{tabular}{llrrrl}
\hline No. & Nombre & Valor Capital & $\Delta$ Div por $1 \%$ margen & Valor Capital & $\begin{array}{l}\Delta \% \text { Valor } \\
\text { Capital }\end{array}$ \\
\hline 1 & $\begin{array}{l}\text { Banco Del } \\
\text { Bajío }\end{array}$ & 54,951 & 697.49 & $63,091.24$ & $14.81 \%$ \\
\hline 2 & GFBanorte & 390,132 & $7,399.27$ & $407,229.95$ & $4.38 \%$ \\
\hline 3 & GFInbursa & 195,009 & $2,667.05$ & $199,134.07$ & $2.12 \%$ \\
\hline 4 & Regional & 38,624 & 452.32 & $40,231.51$ & $4.16 \%$ \\
\hline 5 & $\begin{array}{l}\text { Santander } \\
\text { Serfin GF }\end{array}$ & 197,773 & $10,012.33$ & $207,734.58$ & $5.04 \%$ \\
\hline
\end{tabular}

Fuente: elaborado con datos de Economática.

Por último, es evidente que, al relacionar el valor de la empresa por un mayor crecimiento, contra el valor de la empresa por un mayor margen, resulta en un 
mayor número de veces el valor de la empresa por incremento del crecimiento que por el aumento de su margen, similar a la métrica de valor relativo de crecimiento desarrollada por Mass (2005), el sector financiero bancario priorizará la aplicación y desarrollo de las Fintech al crecimiento (ver tabla 8).

CUADRO 8. RELACIÓN DEL VALOR POR CRECIMIENTO VERSUS EL VALOR POR RENTABILIDAD

\begin{tabular}{llrrr}
\hline \multirow{2}{*}{ No. } & Nombre & \multicolumn{2}{c}{ Valor del Capital por } & Valor por crec. / \\
\cline { 3 - 5 } & & mayor crecimiento & mayor margen & Valor por margen \\
\hline 1 & Banco Del Bajío & $292,324.64$ & $63,091.24$ & 6.91 \\
\hline 2 & GFBanorte & $773,371.71$ & $407,229.95$ & 1.79 \\
\hline 3 & GFInbursa & $614,445.92$ & $199,134.07$ & 1.75 \\
\hline 4 & Regional & $191,928.96$ & $40,231.51$ & 6.34 \\
\hline 5 & Santander Serfin GF & $247,099.99$ & $207,734.58$ & 1.15 \\
\hline
\end{tabular}

Fuente: elaborado con datos de Economática.

Nótese cómo el valor por mayor crecimiento de las empresas representa más de seis veces el valor por rentabilidad en los casos de Banco del Bajío y Regional; mientras que, para las demás empresas, representa menos de dos veces por rentabilidad. Cabe señalar cómo la diferencia del valor por crecimiento y el valor por margen es menor en Santander, casi igualando el valor que obtendría por más crecimiento o por mayor rentabilidad.

\section{Conclusiones}

Tanto el incremento en la tasa de crecimiento como en la rentabilidad generan un mayor valor de las empresas en el mercado. En el total de la muestra de grupos financieros analizados, el incremento en el crecimiento a perpetuidad de los flujos de dividendos generó un mayor valor del capital de los accionistas que el incremento en la rentabilidad por el margen neto. Esta situación sugiere que las estrategias a través de las Fintech deberían estar orientadas a un mayor incremento de la oferta de servicios y productos financieros para aprovechar el potencial de bancarización en inclusión financiera que ofrece el mercado mexicano. Ello implicaría que todavía hay potencial de crecimiento y que las instituciones financieras 
todavía no se encuentran totalmente maduras o desarrolladas y/o en un mercado maduro o desarrollado.

Se confirman los fundamentos planteados por Damodaran (2006) en el que sostiene que los pilares o elementos para generar valor son el crecimiento y rentabilidad, en este caso siendo el crecimiento el de mayor impacto o importancia. Así como los hallazgos de Mass (2005) en lo relativo a las diferencias significativas de valor tanto en estrategias de crecimiento como de rentabilidad en empresas norteamericanas, cabe señalar que sus resultados en instituciones o entidades financieras también indicaban que el crecimiento generaría un mayor valos en dichas empresas. Es importante concluir que las empresas deben estimar el valor generado por el crecimiento y la rentabilidad, así como su equivalencia en la generación de valor al momento de establecer sus estrategias y objetivos financieros a largo plazo.

\section{Bibliografía}

Adam, J., (2005). Análisis de los métodos de valuación de empresas y la relación de estos con la capacidad que las organizaciones tienen para generar valor económico: propuesta para reportar, en la información financiera, el valor de las organizacionesy su capacidad para generarlo. Tesis de doctorado en ciencias de la administración, FCA-UNAM, México.

Arditti, F. y Levy, H. (1977). The weighted average cost of capital as a cutoff rate: a critical examination of the classical texbook weigthed average, Financial management (fall), pp. 24-34.

Copeland T., Koller, T. y Murrin, J. (2000). Valuation: measuring and managing the value of companies, third edition, John Wiley \& Sons, New Jersey.

Damodaran, A. (2006). Damodaran on valuation, John Wiley \& Sons, New Jersey.

Drucker, P. (1995). The information executives truly need, Harvard Business Review, Boston.

Entzana C. (2016). Estrategias financieras para crear valor en las empresas que integran el sector detallista y que cotizan en la Bolsa Mexicana de Valores: crecimiento en ventas vs margen de rentabilidad. Tesis para obtener el grado de Maestra en Finanzas, FCA-UNAM, D.F.

Fernández, P., (2013). Valoración de empresas: como medir y gestionar la creación de valor. tercera edición, Cestión 2000, Barcelona. 